\title{
De-noising of dynamic magnetic resonance images by the combined application of wavelet filtering and Karhunen-Loeve Transform (KLT)
}

\author{
Prashanth Palaniappan ${ }^{1 *}$, Orlando P Simonetti ${ }^{2}$, Yu Ding ${ }^{2}$ \\ From 15th Annual SCMR Scientific Sessions \\ Orlando, FL, USA. 2-5 February 2012
}

\section{Background}

Real-time dynamic magnetic resonance imaging (MRI) typically sacrifices signal-to-noise ratio (SNR) to achieve higher spatial and temporal resolution. Spatial and/or temporal filtering methods can be used increase SNR, but at the expense of edge sharpness, i.e., image blurring.

\section{Objective}

In our current study, we describe a new approach to filtering dynamic image series that aims to remove noise without blurring stationary/ moving edges and requires no training data.

\section{Methods}

The proposed approach combines 2D spatial wavelet filtering with 1D temporal Karhunen-Loeve Transform (KLT). The KLT is first applied to create a series of "eigenimages" in which important signal information is concentrated into only a few eigenimages. Then a 2D spatial wavelet filter is applied to each of the individual eigenimages. An adaptive threshold is used to define the wavelet filter strength for each of the eigenimages based on the noise variance and standard deviation of the signal, resulting in stronger filtering of the eigenimages that primarily contain noise. After wavelet filtering, the de-noised eigenimages are transformed back into image space.

The performance of this filtering approach was tested over a range of wavelet filter strengths ( $1 \mathrm{x}$ to $4 \mathrm{x}$ adaptive threshold) for noise reduction and edge sharpness in a digital phantom. SNR improvement was also

${ }^{1}$ Department of Electrical and Computer Engineering, The Ohio State

University, Columbus, OH, USA

Full list of author information is available at the end of the article evaluated in real-time stress cine image series acquired in five normal volunteers. SNR was calculated in phantom and human images by finding the mean signal value and noise variance in a region-of-interest (ROI) within each frame. The sharpness in phantom images was measured as the distance between $20 \%$ and $80 \%$ of the total rise/fall for a moving edge.

\section{Results}

For the digital phantom images, it is evident from the SNR plot that the proposed algorithm gives better results compared to 3 other filtering techniques- spatial wavelet filtering alone, temporal KLT filtering and spatial Gaussian 2D low pass filtering.

From sharpness measurements done on the phantom images, it was observed that the proposed algorithm preserves stationery edge sharpness better compared to spatial filters (wavelet and Gaussian) and preserves moving edge sharpness better compared to temporal filters (KLT). For real-time stress cine images, the SNR improvement was $61.75 \%$ for wavelet threshold*1, $78.07 \%$ for threshold $* 2$ and $94.65 \%$ for threshold $* 3$.

\section{Conclusions}

Noise reduction in real-time cine images of up to $62 \%$ $+/-17 \%$ is feasible without visible edge blurring using the proposed method of spatial wavelet filtering in the temporal eigenimage domain. Future studies will investigate the application of this method in other SNR-challenged dynamic acquisitions such as first-pass perfusion and real-time phase contrast.

\section{Funding}

R01HL102450. 


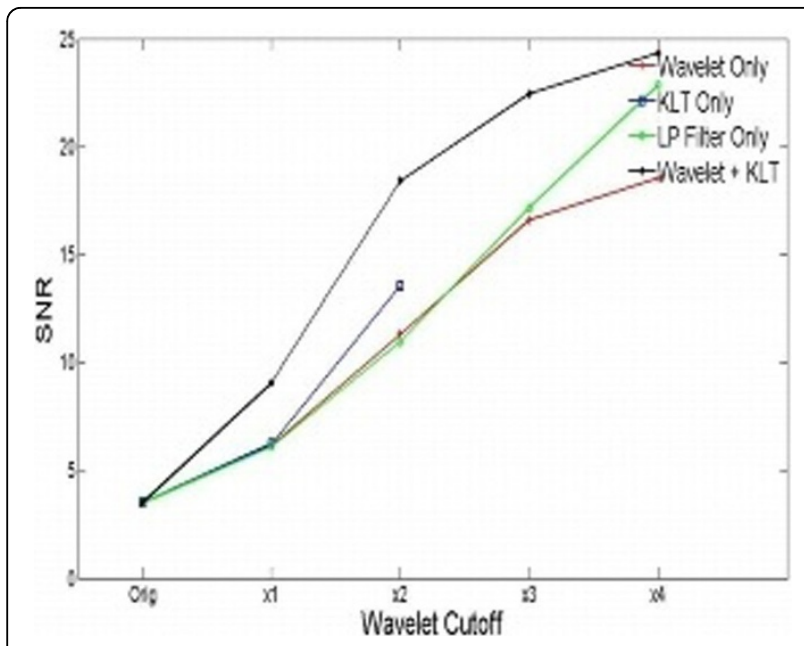

Figure 1 SNR characteristics of different filtering techiques for digital phantom data

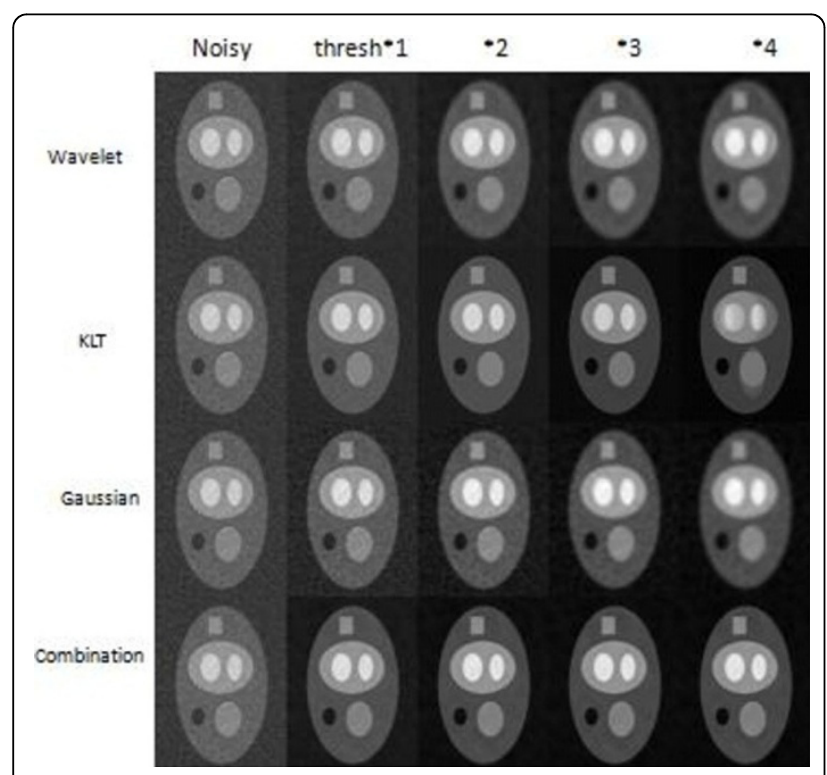

Figure 2 Performance of different filtering techniques

\section{Author details}

'Department of Electrical and Computer Engineering, The Ohio State University, Columbus, OH, USA. ${ }^{2}$ The Ohio State University, Columbus, $\mathrm{OH}$, USA.

Published: 1 February 2012

\section{doi:10.1186/1532-429X-14-S1-W71}

Cite this article as: Palaniappan et al.: De-noising of dynamic magnetic resonance images by the combined application of wavelet filtering and Karhunen-Loeve Transform (KLT). Journal of Cardiovascular Magnetic Resonance 2012 14(Suppl 1):W71.

\section{Submit your next manuscript to BioMed Central} and take full advantage of:

- Convenient online submission

- Thorough peer review

- No space constraints or color figure charges

- Immediate publication on acceptance

- Inclusion in PubMed, CAS, Scopus and Google Scholar

- Research which is freely available for redistribution

Submit your manuscript at www.biomedcentral.com/submit 